\title{
Cement-free Binders for Radioactive Waste Produced from Blast-furnace Slag using Vortex Layer Activation Technology
}

\author{
Ilya Mazov ${ }^{1,2}$, Bekzod Khaydarov ${ }^{1, *}$, Dmitrii Suvorov ${ }^{1}$, Tamara Yudintseva ${ }^{1}$, Denis Kuznetsov ${ }^{l}$, Andrey Yudin $^{1}$ \\ ${ }^{1}$ National University of Science and Technology "MISIS", Moscow, Russian Federation \\ ${ }^{2}$ National Research Tomsk State University, Tomsk, Russian Federation
}

\begin{abstract}
The paper addresses the issue of recycling granulated blast-furnace slag (gBFS) as a source for production of cement-free binder materials for further usage in rare-earth metals production for radioactive waste disposal. The use of the vortex layer activator was provided as main technique allowing to produce high-dispersed chemically activated binders. The paper examines the effect of processing conditions on the physical-chemical and mechanical properties of the resulting BFS-based cement-free materials and gBFSbased concretes.
\end{abstract}

\section{Introduction}

Radioactive waste is a major problem in the production of rare-earth metals. Burial thorium and its products are extremely important. The easiest way - is cementing slurry containing all the radionuclides, which are contained in the feedstock, with burial in earthen trenches. The disadvantage of this method is significantly higher consumption of expensive cement. The use of waste iron and steel industry may reduce the cost of the process [1].

Typical industrial metallurgical production results in formation of large amount of technological wastes - ash, slag etc. Steelmaking produces as much slag as 200$1000 \mathrm{~kg}$ per ton of steel. Large amounts of steel slag are wasting soil and water, resulting in high threat to natural resources. Typical yearly production of steel slag in Russia is 5-7 Mton, which makes this problem very important [2].

Blast-furnace slag (BFS) is usually treated in molten state, producing so-called granulated blast-furnace slag (gBFS), containing 3-5 wt. \% of metal inclusions [3]. Granulated BFS usually contains certain amount of alumina, silica and calcium oxide, thus making it perspective source for production of construction materials. Production of high quality cement-free binders using gBFS as a source shows several advantages - such as reduction of ecological threats, usage of local source for road and civilian building etc $[4,5]$.

One of the most difficult problems in this area is improvement of energy effectiveness of the production process of mechanically activated high-dispersive materials, which are needed for production of highstrength binders [6, 7]. Traditional methods, such as hummer mills or ball milling are characterized by high energy consumption and periodical process [8].

The main aim of the work is developing of energy efficient technology for production of clinker-free materials (CFM) and CFM-based concretes by vortex layer mechanical activation using gBFS as source materials [9] and investigation of mechanical properties of produced materials depending on preparation conditions.

\section{Methods and materials}

Granulated BFS was produced by JSC "Severstal" (Russia). This type of BFS is typical for most large (producing more than 3 Mtons of metal per year) metallurgical companies of Russia. Commercial sample of the Portland cement M400 was used as comparative sample.

All chemicals used in this work were purchased locally and are reagent grade or higher.

Investigation of phase and chemical composition was performed using X-ray fluorescent spectrometer ARL 9900 (Thermo Fisher Scientific, USA), providing standard-free analysis.

Particle size distribution was investigated using Analysette 22 NanoTec (Fritsch, Germany). Mean particle size and size distribution was calculated using standard integrated software of the analyzer.

Specific surface area of all samples was measured using Quantachrome NOVA 1200 Surface Area Analyzer (Quantachrome Instruments, USA).

Phase composition of gBFS (both pristine and chemically modified) and corresponding concretes (after natural hardening and hydro thermal treatment (HTT)) were investigated using XRD method. X-ray diffraction measurements were performed using X-ray diffractometer Difray 401 (Scientific Instruments, Russia).

Mechanical properties of concretes were investigated for cubic samples with dimensions $2 \times 2 \times 2 \mathrm{~cm}^{3}$ using Instron $150 \mathrm{LX}$ universal testing machine (Instron, USA).

* Corresponding author: bekzod1991@mail.ru 
Vortex-layer activator (VLA) was used for mechanical and chemical activation of slag. VLA setup consists of electromagnetic coil (inductor) placed in water-cooling hull. Stainless steel beaker is placed inside the coil and filled with material to treat and magnetic (steel) milling elements. In this work we used stainless steel cylinder with size $\varnothing 2 \times 15 \mathrm{~mm}^{3}$. VLA setup allows to perform continuous treatment and is easy scalable.

Two main parameters of the activation process determine properties of the slag, activated using VLA apparatus. These are treatment time and "slag:grinder" ratio. Typical physical-chemical properties such as specific surface area, particle size distribution and morphology of particles were investigated under variation of treatment parameters. Comparative study of properties was performed for pristine commercial Portland cement sample.

Samples of gBFS-based concretes were prepared using mechanically activated slag. Chemically modified samples were prepared using $\mathrm{NaOH}$. Solid base (7 wt.\%) was added to slag before mechanical activation.

Comparative study was performed using concrete samples prepared by mixing of slag or Portland cement with water, typical "water/binder" ratio was set to 0.22 . Note that all concrete samples were investigated after 28 days of hardening.

\section{Results and Discussion}

\subsection{Chemical and Phase Composition of gBFS}

Chemical composition of pristine gBFS is shown in the Table 1. According to chemical composition of the slag its main parameters were calculated, allowing to conclude that the slag sample is mainly basic (Base module $\mathrm{MB}=1.19$, Base coefficient $\mathrm{KB}=1.15$, Activity Index MA $=3.19$, Quality coefficient $K Q=1.59$ ).

Table 1. Chemical composition of gBFS.

\begin{tabular}{|c|c|}
\hline Compound & Amount, at. \% \\
\hline $\mathrm{Na}_{2} \mathrm{O}$ & 0.28 \\
\hline $\mathrm{MgO}$ & 12.18 \\
\hline $\mathrm{Al}_{2} \mathrm{O}_{3}$ & 8.91 \\
\hline $\mathrm{SiO}_{2}$ & 34.85 \\
\hline $\mathrm{P}_{2} \mathrm{O}_{5}$ & 0.01 \\
\hline $\mathrm{K}_{2} \mathrm{O}$ & 0.43 \\
\hline $\mathrm{CaO}$ & 40.03 \\
\hline $\mathrm{TiO}_{2} \mathrm{O}$ & 1.06 \\
\hline $\mathrm{MnO}$ & 0.12 \\
\hline $\mathrm{Fe}_{2} \mathrm{O}_{3}$ & 1.22 \\
\hline
\end{tabular}

As one can see from the Table 1, the content of inactive impurities (iron and manganese oxides) is low.

Chemical and phase composition of gBFS corresponds to that of typical cement clinker materials, allowing one to use it as a source for preparation of clinker-free binders.

\subsection{Influence of activation conditions on slag properties}

Results of particle size and SSA (specific surface area) measurement for gBFS, treated in various conditions, are shown in Table 2. Typical SEM images of activated samples are shown on the Fig. 1.

Table 2. Mean size and specific surface area of gBFS treated in various conditions.

\begin{tabular}{|c|c|c|c|c|c|c|}
\hline \multirow{3}{*}{ 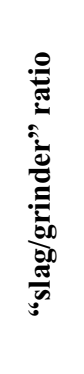 } & \multicolumn{6}{|c|}{ Treatment time } \\
\hline & \multicolumn{2}{|c|}{$3 \mathrm{~min}$} & \multicolumn{2}{|c|}{$5 \mathrm{~min}$} & \multicolumn{2}{|c|}{$7 \mathrm{~min}$} \\
\hline & 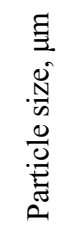 & 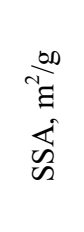 & 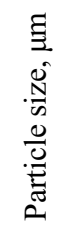 & $\begin{array}{l}\text { DD } \\
\text { ¿ } \\
\text { \& } \\
\text { D }\end{array}$ & 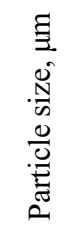 & 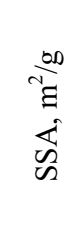 \\
\hline $1: 2$ & 10.8 & 1.509 & 8.0 & 2.244 & 8.5 & 3.267 \\
\hline $1: 2.5$ & 27.6 & 1.454 & 16.7 & 2.256 & 9.7 & 2.154 \\
\hline $1: 3$ & 38.9 & 1.39 & 26.6 & 1.362 & 14.2 & 2.489 \\
\hline $1: 3.5$ & 47.3 & 0.61 & 10.9 & 1.379 & 22.6 & 1.917 \\
\hline
\end{tabular}

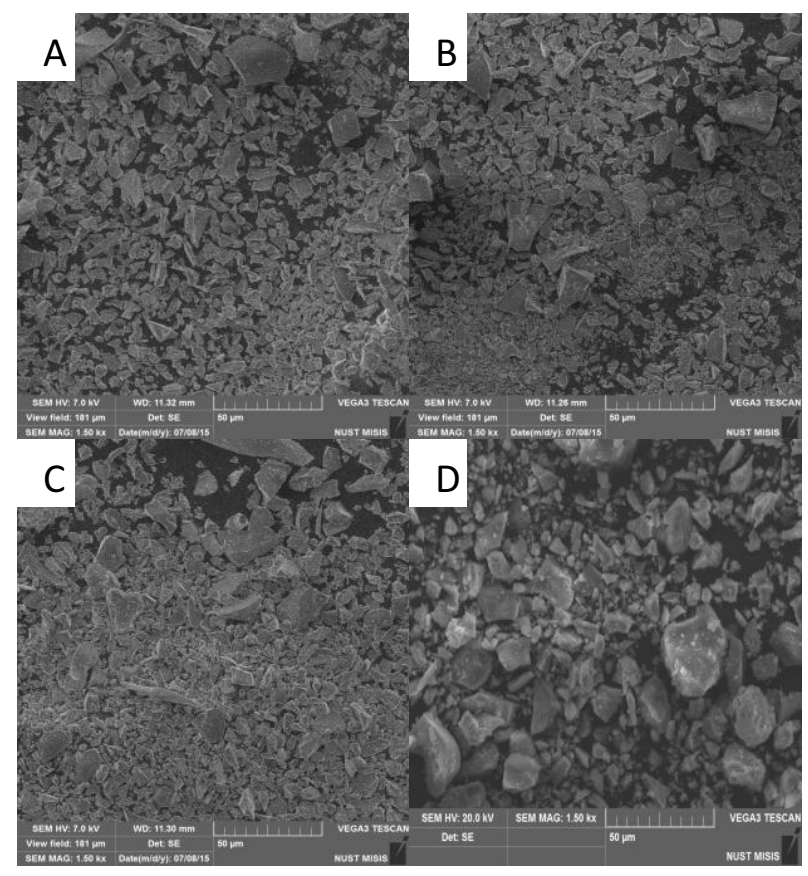

Fig. 1. SEM micrographs of activated gBFS $(3 \mathrm{~min})$ with different "slag/grinder" ratio (A - 1:2, B - 1:3, C - 1:3.5, D pristine Portland cement).

According to the data of Table 2 and Fig. 1 it is possible to conclude that increase of the amount of grinding media in VLA setup results in increase of the mean particle size and in lowering of the specific surface area. This phenomenon most probably occurs due to space limitations in activation chamber and following decrease of the activation efficiency and agglomeration of the slag particles. This is due to increase of number of grinder-to-grinder impacts instead of grinder-to-slag impacts. Thus optimal "slag/grinder" ratio is 1:2 ...1:2.5. 
Increase of the treatment period results in simultaneous increase of the specific surface area and diminishing of the slag particles.

Taking into account above mentioned considerations, following conditions of VLA treatment were chosen as optimal: 5 minutes treatment time with "slag/grinder" ratio 1:2. Treatment in such conditions allows to produce activated gBFS with particle size 10-15 $\mu \mathrm{m}$ and SSA $=$ $2.2 \ldots 3.2 \mathrm{~m}^{2} / \mathrm{g}$.

Properties of the commercial sample of Portland cement were also investigated. Typical SSA value is $1.7 \ldots 1.9 \mathrm{~m}^{2} / \mathrm{g}$ and mean particle size is $14 \ldots 16 \mu \mathrm{m}$. Thus mechanically activated gBFS shows structural properties close to that of Portland cement and the main issue, determining its possible application as a clinkerfree binder, is phase composition of pristine materials and corresponding concretes.

\subsection{Phase composition of gBFS and corresponding concretes}

XRD data for both Portland cement and PC-based concrete shows high amount of alite (C3S) and belite (C2S) phases (Fig.2). In contrast, pristine g-BFS is almost amorphous and no significant peaks can be found on XRD spectrum.

Certain amount of crystal (or semi-crystal) alite phase can be found in VLA-treated gBFS sample. The same peak (of hydrated alite phase) can be found on the $\mathrm{X}$-ray diffractogram of gBFS-based concrete. However, amount of this phase is relatively low and it should be raised in order to improve mechanical properties of concrete.

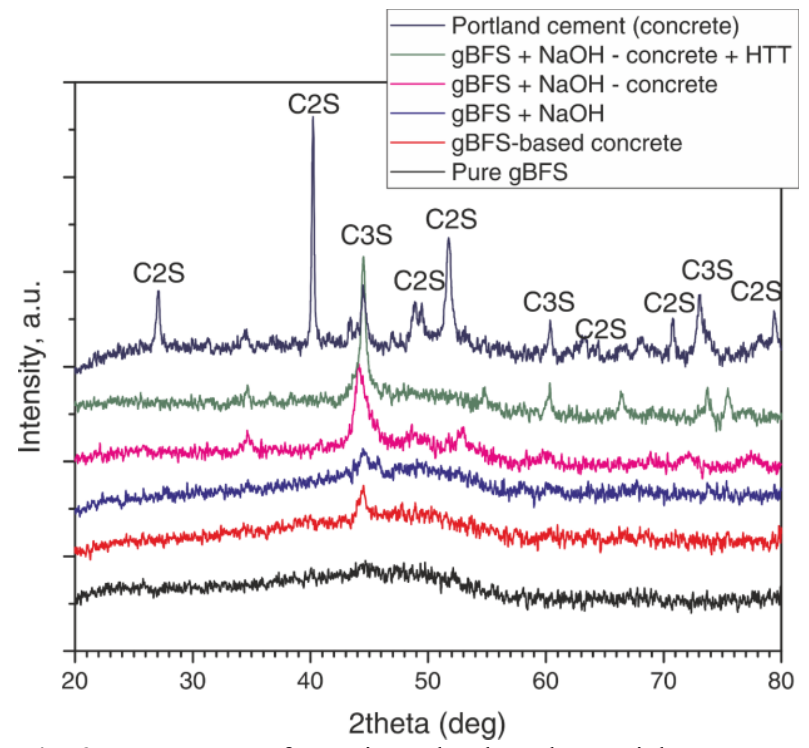

Fig. 2. XRD spectra for various slag-based materials.

Addition of $\mathrm{NaOH}$ to the gBFS during VLA treatment results in significant increase of amount of crystal phases both in slag and slag-based concrete. Still amount of the crystal phase is low as compared with Portland cement and PC-based concrete.

Hydrothermal treatment of NaOH-modified gBFSbased concrete results in further increase of the amount of alite and belite phases thereby it is comparable with that of PC-based concrete.

\subsection{Mechanical properties of concretes}

Results of investigation of mechanical properties of PCbased and gBFS-based concretes are shown in the Table 3. According to the presented data it can conclude that mechanical properties of gBFS-based concretes are strictly dependent on phase composition and on mechanical and chemical activation as well as on posthardening treatment of the concrete.

The highest strength value was achieved for the sample of $\mathrm{NaOH}$-activated g-BFS which was hydrothermally treated after hardening. This sample shows the highest amount of crystal phases of XRD spectra and is comparable with PC sample.

Table 3. Mechanical properties of PC-based and gBFS-based concretes.

\begin{tabular}{|l|l|}
\hline Sample & Strength \\
\hline PC M400 & $39.5 \mathrm{MPa}$ \\
\hline gBFS w/o chemical modification & $12.1 \mathrm{MPa}$ \\
\hline gBFS $+7 \% \mathrm{NaOH}$ w/o post-treatment & $38.7 \mathrm{MPa}$ \\
\hline gBFS $+7 \% \mathrm{NaOH}+$ HT treatment & $44.7 \mathrm{MPa}$ \\
\hline
\end{tabular}

Energy efficiency of VLA approach was calculated in comparison with traditional ball milling technology. In order to produce activated gBFS with high specific surface area $\left(1.5-3.2 \mathrm{~m}^{2} / \mathrm{g}\right)$ one needs to apply VLA activation for 3-7 minutes, and at the same time ball milling approach needs several hours to achieve same parameters. Energy consumption depends strongly on the power of the mill used and can be estimated as 3-5 times higher for ball mill as compared with VLA setup.

\section{Conclusion}

Clinker-free cementitious binders were prepared using granulated blastfurnace slag as a source material. Novel vortex layer activation approach was used for preparation of mechanically and chemically activated materials.

Structural and mechanical properties of slag-based materials were investigated. Such properties' depending on preparation conditions as well as optimal activation conditions were determined.

Mechanical properties of slag-based concretes were investigated depending on preparation conditions and post-hardening treatment. It was shown that strength of $\mathrm{NaOH}$-activated slag-based concretes with hydrothermal treatment is outreaching that of PC-based concretes (44.7 $\mathrm{MPa}$ and $39.5 \mathrm{MPa}$, correspondingly). It makes slagbased materials perspective for burial of rare-earth and radioactive wastes.

Presented work indicate that the developed method of clinker-free cement producing may be suitable for the production of binder materials for the recycling and burial of rare and radioactive earth metals waste disposal. The use of this material will simplify the 
technology and reduce costs due to the use of metallurgical production waste.

Authors are kindly grateful for support of this work by Ministry of Science and Education of Russian Federation (project RFMEFI57814X0099).

\section{References}

1. R. Ausness, Wis. L. Rev. 707 (1979)

2. J. Zelic, Cement Concrete Res. 35, 2340 (2005)

3. S. Bostanc1, M. Limbachiya, H. Kew. J. Cleaner Production 112(1), 542 (2016)

4. W.T. Kuo, H.Y. Wang, C.Y. Shu. Construction and Build. Mater., 63, 189 (2014)

5. I. Ismail, A. S. Bernal, J. L. Provis, e.a. Cement and Concrete Composites, 45, p. 125-135 (2014)

6. A.S. Bernal, R.M. de Gutiérrez, L.J. Provis, Construction and Build. Mater., 33, 99 (2012)

7. X. Huang, Z. Wang, Y. Liu, W. Hu, W. Ni. Construction and Build. Mater. 112, 241 (2016)

8. J.I.E. Garcia, J.M. Nonell, A. Gorokhovsky, Bol. Soc. Esp. Ceram. Vidrio, 41(5), 451 (2002)

9. A.V. Gorokhovsky, J.I. Escalante-Garcia, E. Sanchez-Valdes, I.N. Burmistrov, D.V. Kuznetsov, Ceramics Int. 41, 13294 (2015) 